\title{
MEROMORPHIC FUNCTIONS OF BOUNDED VALENCE ON AN OPEN RIEMANN SURFACE
}

\author{
Fumio Maitani and Hisashi Ishida
}

\section{Introduction}

In the theory of the boundary behaviour of canonical conformal mappings on open Riemann surfaces, it is a well known fact that every canonical conformal mapping on an open Riemann surface of finite genus is a vertical slit mapping ([4], [6], [9]). This theorem was first proved by M. Mori ([7]). For the proof of the theorem, she prepared a lemma (Lemma 2, p. 177) which asserts that every meromorphic function $f$ of bounded valence on an open Riemann surface $R$ of finite genus has a limit at each weak boundary point of the Kerékjártó-Stoilow boundary of $R$.

However, P. Järvi ([3]) showed that the assertion of the lemma contains invalid arguments. Although he could not restore the assertion of Mori, he proved that the lemma holds true if $R$ is an open Riemann surface with absolutely disconnected boundary (see [8]) and if no cluster set of $f$ at Kerékjártó-Stoillow boundary point of $R$ separates the extended complex plane. He further pointed out an error in the example introduced by Kusunoki and Taniguchi ([5]) who intended to show that $\cap_{n=1}^{\infty} O_{A D, n}-O_{A D} \neq \varnothing$. Moreover, he remarked that one can obtain a planar Riemann surface of the class $\cap_{n=1}^{\infty} O_{A D, n}-O_{A D}$ provided that Mori's assertion holds true for planar Riemann surfaces.

In this note, we shall first give a simple example of a Riemann surface to show that Mori's assertion does not hold even for planar Riemann surfaces.

Secondly, we shall prove that every meromorphic function of bounded valence on an open Riemann surface $R$ with absolutely disconnected boundary admits a continuous extension to the Kerékjártó-Stoillow boundary of $R$. Consequently, according to the proof of Järvi ([3]), we can conclude that there exists a planar Riemann surface which belongs to the class $\cap_{n=1}^{\infty} O_{A D, n}-O_{A D}$.

\section{The boundary behaviour of meromorphic functions of bounded valence at weak boundary points}

Let $R$ be an open Riemann surface and $\Gamma$ be a family of locally rectifiable curves $\gamma$ in $R$. Consider the class of Borel measurable linear densities $\varrho|d z|$ on $R$ for which the quantities

$$
L(\Gamma, \varrho)=\inf \left\{\int_{\gamma} \varrho|d z| ; \gamma \in \Gamma\right\}, \quad A(\varrho, R)=\iint_{R} \varrho^{2} d x d y
$$


are well-defined and not simultaneously 0 or $\infty$. Then the quantity

$$
\lambda(\Gamma)=\sup \left\{\frac{L(\Gamma, \varrho)^{2}}{A(\varrho, R)} ; \varrho|d z|\right\}
$$

is called the extremal length of $\Gamma$. Let $R^{*}$ be the Kerékjártó-Stoillow compactification of $R$ and $\beta$ be the Kerékjártó-Stoilow boundary of $R$. Let $p$ be a point in $\beta$ and $V$ be a subregion of $R$ whose relative boundary $\partial V$ is compact and regular. We say that $V$ is an end of $p$ if the closure of $V$ in $R^{*}$ contains $p$. Let $\Gamma(p, V)$ be the family of cycles $\gamma$ in $V$ separating $p$ and $\partial V$. We say that a point $p \in \beta$ is weak or a weak boundary point if, for any end $V$ of $p$, the extremal length $\lambda(\Gamma(p, V))$ of $\Gamma(p, V)$ equals 0 .

For a meromorphic function $f$ on $R$ and $p \in \beta$ we call

$$
\mathrm{Cl}(f, p)=\cap\{\overline{f(V)} ; V \text { is an end of } p\}
$$

the cluster set of $f$ at $p$. We say that $f$ has a limit at $p$ if $\mathrm{Cl}(f, p)$ consists of one point.

Mori ([7]) asserts in Lemma 2 that every meromorphic function $f$ of bounded valence on an open Riemann surface $R$ of finite genus has a limit at each weak boundary point of the Kerékjártó-Stoïlow boundary of $R$. However, P. Järvi ([3]) pointed out that the proof contains incorrect arguments. In fact, we can give a counter-example.

Take a decreasing sequence $\left\{a_{n}\right\}_{n=1}^{\infty}$ of positive numbers such that $\lim _{n \rightarrow \infty} a_{n}$ $=0$ and $\sum_{n=1}^{\infty} \log \left(a_{2 n-1} / a_{2 n}\right)=\infty$. Take another sequence $\left\{b_{n}\right\}_{n=2}^{\infty}$ such that $a_{2 n+1}<b_{2 n+1}<b_{2 n}<a_{2 n}$. Delete a countable number of closed intervals $\left[b_{2 n+1}, b_{2 n}\right](n=1,2, \ldots)$ on the real axis from the punctured extended complex plane $\{0<|z| \leq \infty\}$. Denote the remaining region by $R_{1}$. Take a countable number of rectangles without closed intervals on the real axis and denote them by

$$
B_{n}=\left\{z ; a_{2 n+1}<\operatorname{Re}(z)<a_{2 n},-1<\operatorname{Im}(z)<1\right\}-\left[b_{2 n+1}, b_{2 n}\right]
$$

$(n=1,2, \ldots)$. For each $n(n=1,2, \ldots)$, join $R_{1}$ with $B_{n}$ crosswise along the slit $\left[b_{2 n+1}, b_{2 n}\right]$. By this construction we obtain a two-sheeted covering surface $R$ over the extended complex plane. It is easy to see that $R$ is a planar Riemann surface. Let $f$ be the projection map from $R$ to the extended complex plane. Then $f$ is a meromorphic function of 2-valence on $R$. Let $p$ be the Kerékjártó-Stoïlow boundary point over 0 . Then $f$ has not a limit at $p$. In fact, for any end $V$ of $p$, there is an $n$ such that $V \supset \cup_{k=n}^{\infty} B_{k}$. Hence the cluster set of $f$ at $p$ contains a proper continuum $\{$ iy $;-1 \leq y \leq 1\}$.

On the other hand, we can prove that $p$ is a weak boundary point as follows. Let $V$ be an arbitrary end of $p$ and $A_{n}$ be the annulus in $R_{1}$ lying over the annulus $\left\{z ; a_{2 n}<|z|<a_{2 n-1}\right\} \quad(n=1,2, \ldots)$. Let $\Gamma_{n}$ be the family of curves 
$\gamma_{r}$ in $A_{n}$ lying over the circles $\{z ;|z|=r\}$, where $a_{2 n}<r<a_{2 n-1}$. Then there exists an $n$ such that $\cup_{k=n}^{\infty} \Gamma_{k} \subset \Gamma(p, V)$. Since

$$
\frac{1}{\lambda(\Gamma(p, V))} \geq \sum_{k=n}^{\infty} \frac{1}{\lambda\left(\Gamma_{k}\right)}=\frac{1}{2 \pi} \sum_{k=n}^{\infty} \log \left(a_{2 k-1} / a_{2 k}\right)=\infty
$$

$\lambda(\Gamma(p, V))=0$. Thus $p$ is a weak boundary point.

\section{Cluster sets of meromorphic functions of bounded valence}

Let $R$ be an open Riemann surface and $f$ a meromorphic function of bounded valence on $R$. We call

$$
\mathrm{Cl}(f, \beta)=\cap\{\overline{f(R-K)} ; K \text { is a compact set in } R\}
$$

the cluster set of $f$ at the Kerékjártó-Stoilow boundary $\beta$ of $R$.

Lemma 1. Let $f$ be a meromorphic function of bounded valence on $R$. Then $\mathrm{Cl}(f, \beta)$ is nowhere dense in the extended complex plane.

Proof. Suppose that $\mathrm{Cl}(f, \beta)$ includes an open disk $D_{0}$. There exist a point $z_{1} \in R$ and a relatively compact neighbourhood $U_{1}$ of $z_{1}$ such that $f\left(U_{1}\right) \subset$ $D_{0}$. Inductively, for each integer $m(m=2,3, \ldots)$ we have points $z_{m} \in R$ and neighbourhoods $U_{m}$ of $z_{m}$ such that $f\left(U_{m}\right) \subset f\left(U_{m-1}\right)$ and $U_{m} \cap\left(\cup_{i=1}^{m-1} U_{i}\right)=\varnothing$. This is contradictory to $f$ being of bounded valence.

Lemma 2. Let $f$ be a meromorphic function of bounded valence on $R$ and $\gamma^{\prime}$ be a component of $\mathrm{Cl}(f, \beta)$ which is a proper continuum. Then, for any open disk $D$ with $D \cap \gamma^{\prime} \neq \varnothing$, there exists a point $p \in \beta$ such that $\mathrm{Cl}(f, p)$ is a proper continuum and $\mathrm{Cl}(f, p) \cap \gamma^{\prime} \cap D \neq \varnothing$.

Proof. Contrary to the assertion, suppose that $\mathrm{Cl}(f, p)$ reduces to a point for any $p \in \beta$ satisfying $\operatorname{Cl}(f, p) \cap \gamma^{\prime} \cap D \neq \varnothing$. There exists a point $p_{1} \in \beta$ such that $\operatorname{Cl}\left(f, p_{1}\right) \cap \gamma^{\prime} \cap D \neq \varnothing$. Since $\operatorname{Cl}\left(f, p_{1}\right)$ is a singleton, denoted by $w_{1}$, we can take an end $V_{1}$ of $p_{1}$ such that $f\left(V_{1}\right) \subset D$ and the unbounded component of $\hat{\mathbf{C}}-f\left(V_{1}\right)$ meets $\gamma^{\prime}$.

Now suppose $f\left(V_{1}\right) \cap \gamma^{\prime}=\varnothing$. There is an end $V_{1}^{\prime}$ of $p_{1}$ such that $V_{1}^{\prime}$ is properly contained in $V_{1}$ and $f\left(\partial V_{1}^{\prime}\right) \cap \gamma^{\prime}=\varnothing$. Then the unbounded component $E$ of $\hat{\mathbf{C}}-f\left(V_{1}^{\prime}\right)$ contains $\gamma^{\prime}$. On the other hand, $f\left(V_{1}^{\prime}\right)$ contains a sequence of points converging to $w_{1}$. Hence the outer boundary $\partial E$ of $f\left(V_{1}^{\prime}\right)$ is contained in $\gamma^{\prime}$. Similarly we should have the other end $V_{1}^{\prime \prime}$ of $p_{1}$ such that $E$ is properly included in the unbounded component of $\hat{\mathbf{C}}-f\left(V_{1}^{\prime \prime}\right)$ and the outer boundary of $f\left(V_{1}^{\prime \prime}\right)$ is contained in $\gamma^{\prime}$. This is contradictory to $f\left(V_{1}\right)$ being connected. Hence, $f\left(V_{1}\right) \cap \gamma^{\prime} \neq \varnothing$. 
Let $w_{2}\left(\neq w_{1}\right) \in f\left(V_{1}\right) \cap \gamma^{\prime}$. Then there exist a relatively compact subregion $U_{1}$ of $V_{1}$ such that $w_{2} \in f\left(U_{1}\right) \subset f\left(V_{1}\right) \subset D$. On the other hand, there is a point $p_{2} \in \beta$ such that $\operatorname{Cl}\left(f, p_{2}\right)=\left\{w_{2}\right\} \subset \gamma^{\prime} \cap D$. Hence there is an end $V_{2}$ such that $f\left(V_{2}\right) \subset f\left(U_{1}\right)$. Therefore there exist a point $w_{3} \in \gamma^{\prime}$ and a relatively compact subregion $U_{2}$ of $V_{2}$ such that $w_{3} \in f\left(U_{2}\right)$ and $U_{1} \cap U_{2}=\varnothing$. Inductively, we have ends $V_{m}$ and relatively compact subregions $U_{m}$ of $V_{m}$ such that $f\left(U_{m}\right) \subset$ $f\left(U_{m-1}\right)$ and $U_{m} \cap\left(\cup_{i=1}^{m-1} U_{i}\right)=\varnothing$. This is contradictory to $f$ being of bounded valence.

\section{Meromorphic function of bounded valence and absolutely disconnected boundary}

Let $R$ be an open Riemann surface and $\beta$ be the Kerékjártó-Stoïlow boundary of $R$. Let $f$ be a meromorphic function of bounded valence. We denote the totality of components of $\hat{\mathbf{C}}-\mathrm{Cl}(f, \beta)$ by $\left\{G_{i}\right\}$. Note that $f(R) \cap G_{i} \neq \varnothing$ implies $f(R) \supset G_{i}$.

Lemma 3. Let $f$ be a meromorphic function of bounded valence on $R$. Suppose that $p$ is a weak boundary point of $\beta$ such that $\mathrm{Cl}(f, p)$ is a proper continuum. Then, for any disk $D$ with $D \cap \mathrm{Cl}(f, p) \neq \varnothing$ there is an infinite number of members $\left\{G_{i_{n}}\right\}$ of $\left\{G_{i}\right\}$ such that $D \cap G_{i_{n}} \neq \varnothing$ and $f(R) \supset G_{i_{n}}$.

Proof. Denote $\mathrm{Cl}(f, p)$ by $\gamma$. We may assume that $\gamma$ is a bounded closed set in C. Take an end $V_{0}$ of $p$ such that $\overline{f\left(V_{0}\right)}$ is a bounded closed set in C. Let $\varrho|d z|$ be the linear density on $V_{0}$ which is the pull-back of the Lebesgue measure on $\mathbf{C}$ by $f$. Since $f$ is of bounded valence on $f\left(V_{0}\right), A\left(\varrho, V_{0}\right)<\infty$ and $L\left(\Gamma\left(p, V_{0}\right), \varrho\right)=0$. Take a point $w_{0} \in \gamma$ and a disk $D\left(w_{0}, \varepsilon\right)=\left\{w ;\left|w-w_{0}\right|<\varepsilon\right\}$ whose boundary meets $\gamma$. By Lemma 1 , there is a member of $\left\{G_{i}\right\}$, say $G_{1}$, such that $D\left(w_{0}, \varepsilon\right) \cap G_{1} \neq \varnothing$ and $f(R) \supset G_{1}$.

Contrary to the assertion, suppose that there is only a finite number of members, say $\left\{G_{i}\right\}_{i=1, \ldots n}$, such that $D\left(w_{0}, \varepsilon\right) \cap G_{i} \neq \varnothing$ and $f(R) \supset G_{i}$. We can choose ends $\left\{V_{k}\right\}$ of $p$ such that $\partial V_{k} \in \Gamma\left(p, V_{0}\right)$ and $\int_{\partial V_{k}} \varrho|d z|<\varepsilon / 2^{k}$. Let $J_{k}$ be the unbounded component (that is, the component including $\infty$ ) of $\hat{\mathbf{C}}-f\left(\partial V_{k}\right)$. Since $f\left(\partial V_{k}\right)$ cannot enclose $\gamma \cap D\left(w_{0}, \varepsilon\right), J_{k}$ contains a point in $\gamma \cap D\left(w_{0}, \varepsilon\right)$. There exist a $G_{i}(1 \leq i \leq n)$ and a component $K_{i}$ of $J_{k} \cap G_{i}$ such that $f\left(V_{k}\right) \supset K_{i}$. We assume that $\partial V_{k}$ is oriented so that $V_{k}$ lies to the left of $\partial V_{k}$. Then $f\left(\partial V_{k}\right)$ is a finite union of oriented closed curves. If the boundary of a component of $J_{k} \cap G_{i}$ contains an oriented subarc $C$ of $f\left(\partial V_{k}\right)$ and the component lies to the left of $C$, then the component is contained in $f\left(V_{k}\right)$. Generally the sheet number of $f\left(V_{k}\right)$ over a component of $\left\{f\left(V_{k}\right)-J_{k}-f\left(\partial V_{k}\right)\right\} \cap G_{i}$ is higher than the winding number of $f\left(\partial V_{k}\right)$ about a point in the component. In other words we can say what follows. Let $K_{i}^{\prime}$ be any other component of $J_{k} \cap G_{i}$. Take $\zeta_{1} \in K_{i}$ and $\zeta_{2} \in K_{i}^{\prime}$. Then we can take an oriented piecewise analytic arc $l$ connecting $\zeta_{1}$ to $\zeta_{2}$ in $G_{i}$. When $l$ crosses $f\left(\partial V_{k}\right)$ once transversally from right to left (or left to right), then 
the sheet number of $f\left(V_{k}\right)$ increases (or decreases) by one. Since $\zeta_{1}$ and $\zeta_{2}$ are not enclosed by $f\left(\partial V_{k}\right)$, the number of times $l$ crosses $f\left(\partial V_{k}\right)$ from right to left is equal to the number of times $l$ crosses from left to right. Hence we can conclude that $\zeta_{2} \in f\left(V_{k}\right)$. Therefore $J_{k} \cap G_{i}$ is contained in $f\left(V_{k}\right)$. There exist $G_{i_{0}}$ $\left(1 \leq i_{0} \leq n\right)$ and a sequence of ends $V_{k_{m}}$ of $p$ such that $J_{k_{m}} \cap G_{i_{0}} \subset f\left(V_{k_{m}}\right)$. Let $W_{0}$ be a closed disk contained in $G_{i_{0}}$. Since $\cap \overline{f\left(V_{k_{m}}\right)}=\gamma$, for every sufficiently large number $k_{m},\left(J_{k_{m}} \cap G_{i_{0}}\right) \cap W_{0}=\varnothing$; hence $J_{k_{m}} \cap G_{i_{0}} \subset G_{i_{0}}-W_{0}$. Since the length of $\partial J_{k}$ is less than $\varepsilon / 2^{k}$,

$$
\left\{\text { area of }\left(G_{i_{0}}-W_{0}\right)\right\} \geq\left\{\text { area of } J_{k_{m}} \cap G_{i_{0}}\right\} \geq\left\{\text { area of } G_{i_{0}}\right\}-\frac{\varepsilon^{2}}{\pi 2^{2 k_{m}+2}} .
$$

This is a contradiction for a sufficiently large number $k_{m}$.

We say that the Kerékjártó-Stoillow boundary $\beta$ of $R$ is absolutely disconnected if every point $p \in \beta$ is weak.

Theorem 1. Let $f$ be a meromorphic function of bounded valence on $R$ and $\beta$ be absolutely disconnected. Then $\mathrm{Cl}(f, \beta)$ is totally disconnected. Especially, $f$ admits a continuous extension to $\beta$.

Proof. Suppose that $\mathrm{Cl}(f, \beta)$ is not totally disconnected. We may assume that $\mathrm{Cl}(f, \beta)$ is a bounded closed set in $\mathrm{C}$. Let $\varrho|d z|$ be the linear density on $R$ which is the pull-back of the Lebesgue measure on $\mathbf{C}$ by $f$ on $R-f^{-1}(\infty)$ and 0 on $f^{-1}(\infty)$. There is a component $G_{i}$ of $\hat{\mathbf{C}}-\mathrm{Cl}(f, \beta)$ such that $f(R) \supset G_{i}$ and there is a boundary component $\gamma_{i}$ of $G_{i}$ which is a proper continuum. Take a disk $D\left(w_{0}, \varepsilon\right)=\left\{w ;\left|w-w_{0}\right|<\varepsilon\right\}$ such that $w_{0} \in \gamma_{i}$ and $\partial D\left(w_{0}, \varepsilon\right) \cap \gamma_{i} \neq \varnothing$.

We prove that there exist $w_{1} \in \gamma_{i} \cap D\left(w_{0}, \varepsilon\right)$ and $z_{1} \in R$ such that $f\left(z_{1}\right)=$ $w_{1}$. Contrary to the assertion, suppose that $f^{-1}\left(\gamma_{i} \cap D\left(w_{0}, \varepsilon\right)\right)=\varnothing$. There is a sequence $\left\{a_{n}\right\}$ in $G_{i}$ which converges to $w_{0}$. Then $\left\{f^{-1}\left(a_{n}\right)\right\}$ does not cluster in $R$. Let $q \in \beta$ be one of the accumulation points of $\left\{f^{-1}\left(a_{n}\right)\right\}$. Then $\mathrm{Cl}(f, q) \ni w_{0}$. Take an end $V_{0}$ of $q$ such that $\overline{f\left(V_{0}\right)}$ is a bounded closed set in C. Since $q$ is weak, there is a sequence of ends $\left\{V_{k}\right\}$ of $q$ such that $\partial V_{k} \in \Gamma\left(q, V_{0}\right)$ and $\int_{\partial V_{k}} \varrho|d z|<\varepsilon / 2^{k}$. By our supposition $f\left(\partial V_{k}\right)$ does not meet $\gamma_{i} \cap D\left(w_{0}, \varepsilon\right)$. Since $f\left(V_{k}\right) \cap G_{i} \neq \varnothing$, by a similar argument in the proof of Lemma 3, $f\left(V_{k}\right) \supset G_{i} \cap$ $\left\{\right.$ the unbounded component of $\left.\hat{\mathbf{C}}-f\left(\partial V_{k}\right)\right\}$. It follows that $\mathrm{Cl}(f, q)$ contains $G_{i}$, which contradicts Lemma 1 . Hence there exist $w_{1} \in \gamma_{i} \cap D\left(w_{0}, \varepsilon\right)$ and $z_{1} \in R$ such that $f\left(z_{1}\right)=w_{1}$. Therefore we have a relatively compact neighbourhood $U_{1}$ of $z_{1}$ and a disk $D\left(w_{1}, \varepsilon_{1}\right)$ such that $f\left(U_{1}\right)=D\left(w_{1}, \varepsilon_{1}\right) \subset D\left(w_{0}, \varepsilon\right)$.

Since $D\left(w_{1}, \varepsilon_{1}\right) \cap \gamma_{i} \neq \varnothing$, by Lemmas 2 and 3 , there is an infinite number of members $\left\{G_{i_{n}}\right\}$ of $\left\{G_{k}\right\}$ such that $D\left(w_{1}, \varepsilon_{1}\right) \cap G_{i_{n}} \neq \varnothing$ and $f(R) \supset G_{i_{n}}$. Since $\overline{f\left(R-U_{1}\right)} \cap D\left(w_{1}, \varepsilon_{1}\right) \neq \varnothing$, we can take $G_{j}(j \neq 1)$ such that $\overline{f\left(R-U_{1}\right)} \supset G_{j} \cap$ $D\left(w_{1}, \varepsilon_{1}\right)$ and $D\left(w_{1}, \varepsilon_{1}\right)$ meets a component $\gamma_{j}$ of $\partial G_{j}$ which is not a point. In the 
same way we can take $w_{2} \in \gamma_{j} \cap D\left(w_{1}, \varepsilon_{1}\right)$ and a relatively compact neighbourhood $U_{2}$ of $w_{2}$ such that $f\left(U_{2}\right)=D\left(w_{2}, \varepsilon_{2}\right) \subset D\left(w_{1}, \varepsilon_{1}\right)$ and $U_{2} \cap U_{1}=\varnothing$. We can repeat this procedure infinitely, which is contradictory to $f$ being of bounded valence.

Remark. We denote by $O_{S B}$ the class of Riemann surfaces which tolerates no univalent bounded analytic function. Let $A D$ be the family of analytic functions with finite Dirichlet integral. We denote by $O_{A D}$ the class of Riemann surfaces on which there are no nonconstant $A D$-functions and by $O_{A D, n}$ the class of Riemann surfaces on which there are no $A D$-functions of at most $n$-valence. Järvi ([3]) asserts that a plane region $G$ in the class $O_{S B}-O_{A D}$ belongs to $\cap_{n=1}^{\infty} O_{A D, n}-$ $O_{A D}$, provided that the assertion of Lemma 2 in Mori [7] holds for planar Riemann surfaces. We have proved in Section 1 that the assertion of the lemma does not hold even for planar Riemann surfaces. However, we know that the Kerékjártó-Stoïlow boundary of a plane region in the class $O_{S B}$ is absolutely disconnected. Hence, we can apply Theorem 1 to restore the proof of Järvi ([3, p. 179]). Accordingly, we conclude that there exists a planar Riemann surface which belongs to the class $\cap_{n=1}^{\infty} O_{A D, n}-O_{A D}$.

\section{References}

[1] Ahlfors, L.V., and AND L. Sario: Riemann surfaces. - Princeton University Press, Princeton, 1960.

[2] Constantinescu, C., and A. Cornea: Ideale Ränder Riemannscher Flächen. - SpringerVerlag, 1963.

[3] J̈̈RVI, P.: On the continuation of meromorphic functions. - Ann. Acad. Sci. Fenn. Ser. A I Math. 12, 1987, 177-184.

[4] KUSUNOKI, Y.: Theory of Abelian integrals and its application to conformal mappings. Mem. Coll. Sci. Univ. Kyoto Ser. A. Math. 32, 1959, 235-258.

[5] Kusunoki, Y., and M. TANiguChI: Remarks on Fuchsian groups associated with open Riemann surfaces. - Ann. Math. Stud. 97, 1981, 377-390.

[6] Mizumoтo, H.: Theory of Abelian differentials and relative extremal length with applications to extremal slit mappings. - Japan. J. Math. 37, 1968, 1-58.

[7] MoRI, M.: Canonical conformal mappings of open Riemann surfaces. - J. Math. Kyoto Univ. 3, 1964, 169-192.

[8] Sario, L., and K. OiKaWa: Capacity functions. - Springer-Verlag, 1969.

[9] Shiba, M.: The Riemann-Hurwitz relation, parallel slit covering map, and continuation of an open Riemann surface of finite genus. - Hiroshima Math. J. 14, 1984, 371-399.

Fumio Maitani

Kyoto Institute of Technology

Department of Mechanical Engineering

Matsugasaki, Sakyoku

Kyoto 606

Japan

Received 25 May 1990
Hisashi Ishida

Kyoto Sangyo University

Department of Mathematics

Kamigamomotoyama, Kitaku

Kyoto 603

Japan 